科 学 通 报

\title{
渗碳体价电子结构及其性质的分析
}

\author{
余瑞璜 张瑞林* 郑伟涛(1) 胡安广(1)
}

（吉林大学物理系,(1)材料科学系,长春 130023)

\section{关链词港碳体 $\mathrm{Fe}, \mathrm{C} 、$ 价电子结构、性质}

参碳体 $\mathrm{Fe}_{3} \mathrm{C}$ 是 $\mathrm{Fe}-\mathrm{C}$ 合金的重要相组分. 对它的研究是研究钢铁材料的基础。其晶体 结构经多人测定结果基本一致,本文采用 Lipson 等 ${ }^{[1]}$ 的结果进行计算.

由于晶体结构的复杂性, 迄今对渗碳体的价电子结构研究的报道尚少, 最近 Häglund 等 人 ${ }^{[2]}$ 用 LMTO 方法对渗碳体 $\mathrm{Fe}_{3} \mathrm{C}$ 的价电子结构及其某些性质作了较深人的讨论,但其中某 些结果并不使人满意. 本文利用余氏固体与分子经验电子理论 ${ }^{[3]}$, 由 $\mathrm{Fe}_{3} \mathrm{C}$ 的晶体结构直接计 算了其价电子结构, 并就此对某些性质作了讨论, 对某些结果还与 Häglund 等的结果作了比 较.

\section{1 晶体结构、实验键距与等同键数}

\section{1 晶体结构}

由 Lipson 和 $\mathrm{Petch}^{[1]}$ 的结果. $\mathrm{Fe}_{3} \mathrm{C}$ 为正交晶系、 $\mathrm{DO}_{11}$ 型结构.空间群 $\boldsymbol{P}_{n m \boldsymbol{m}}(\mathrm{No}: 62)$ 、 $\mathrm{M}=4$. 在 $21^{\circ} \mathrm{C}$ 晶格常数为 $(n m): a=0.50890 ; b-0.67433 ; c-0.45235$. 原子位置及 坐标参数如下:

$$
\begin{array}{cccccc}
\mathrm{FeI} & 4 & c & \cdot m \cdot & x=0.040 ; z=0.833 . \\
\mathrm{FeII} & 8 & d & l & x=0.183 ; y=0.065 ; z=0.333 . \\
\mathrm{C} & 4 & c & \cdot m \cdot & x=0.86 ; \quad z=0.47
\end{array}
$$

\section{2 实验键距}

利用公式:

$$
D_{\mathrm{uv}}-\left\{\left(x_{\mathrm{u}}-x_{\mathrm{v}}\right)^{2} a^{2}+\left(y_{\mathrm{u}}-y_{\mathrm{v}}\right)^{2} b^{2}+\left(z_{\mathrm{u}}-z_{\mathrm{v}}\right)^{2} c^{2}\right\}^{1 / 2} .
$$

各实验键距值 $(\mathrm{nm})$ 如下 (为节省篇幅只列出前 14 个,余可类推):

$$
\begin{aligned}
& D_{\left(n_{\mathrm{A}}\right)}^{\mathrm{Fel}-\mathrm{C}}=0.18564 ; \quad D_{\left(n_{\mathrm{B}}\right)}^{\mathrm{Fel}-\mathrm{C}}=0.18803 ; \\
& D_{\left({ }_{\mathrm{C} C}\right)}^{\mathrm{Pell}-\mathrm{C}}=0.21928 ; \quad D_{\left(n_{\mathrm{D}}\right)}^{\mathrm{Fell}-\mathrm{C}}=0.23139 ; \\
& D_{\left(n_{B}\right)}^{\mathrm{Fell}-\mathrm{C}}=0.20606 ; \quad D_{\left(n_{\mathrm{F}}\right)}^{\mathrm{Pel}-\mathrm{Pell}}=0.25226 ; \\
& D_{\left(n_{\mathrm{G}}\right)}^{\mathrm{Pel}-\mathrm{PeIl}}=0.26720 ; \quad D_{\left(n_{\mathrm{H}}\right)}^{\mathrm{Pel}-\mathrm{Fell}}=0.26835 ;
\end{aligned}
$$

1992-05-30 收稿, 1992-11-28 收修改稿。

- 联系人. 


\section{3 等同链数}

$$
\begin{array}{ll}
D_{\left(n_{\mathrm{I}}\right)}^{\mathrm{Fel}-\mathrm{Fell}}=0.25493 ; & D_{\left(n_{\mathrm{f}}\right)}^{\mathrm{Fel}-\mathrm{Fel}}=0.26530 ; \\
D_{\left(n_{\mathrm{K}}\right)}^{\mathrm{Fell}-\mathrm{Fell}}=0.24950 ; & D_{\left(n_{\mathrm{L}}\right)}^{\mathrm{Fell}-\mathrm{Fell}}=0.26530 ; \\
D_{\left(n_{\mathrm{M}}\right)}^{\mathrm{Fell}-\mathrm{Fell}}=0.25197 ; & D_{\left(n_{\mathrm{N}}\right)}^{\mathrm{Fell}-\mathrm{Fell}}=0.25535 .
\end{array}
$$

由结构式 $\mathrm{Fe}_{3} \mathrm{C} \equiv \mathrm{FeI}_{2} \mathrm{FeIIC}$ ，利用公式 ${ }^{[3]}$ :

$$
I_{a}=I_{\mathrm{M}} I_{s} I_{\mathrm{K}}
$$

求得对应各种键的等同键数 $I_{\alpha}$ 分别为

$$
\begin{aligned}
& I_{\mathrm{A}}=I_{\mathrm{B}}=I_{\mathrm{J}}=I_{\mathrm{K}}=I_{\mathrm{N}}=2, \\
& I_{\mathrm{C}}=I_{\mathrm{D}}=I_{\mathrm{B}}-I_{\mathrm{F}}=I_{\mathrm{G}}=I_{\mathrm{I}}=I_{\mathrm{L}}=I_{L A}=4, \\
& I_{\mathrm{H}}=8 .
\end{aligned}
$$

\section{2 价电子结构计算结果}

表 1 示出了 BLD ${ }^{[3]}$ 计算结果.

表 1 渗碳体 $\mathrm{Fe}_{3} \mathrm{C}$ 的价电子结构

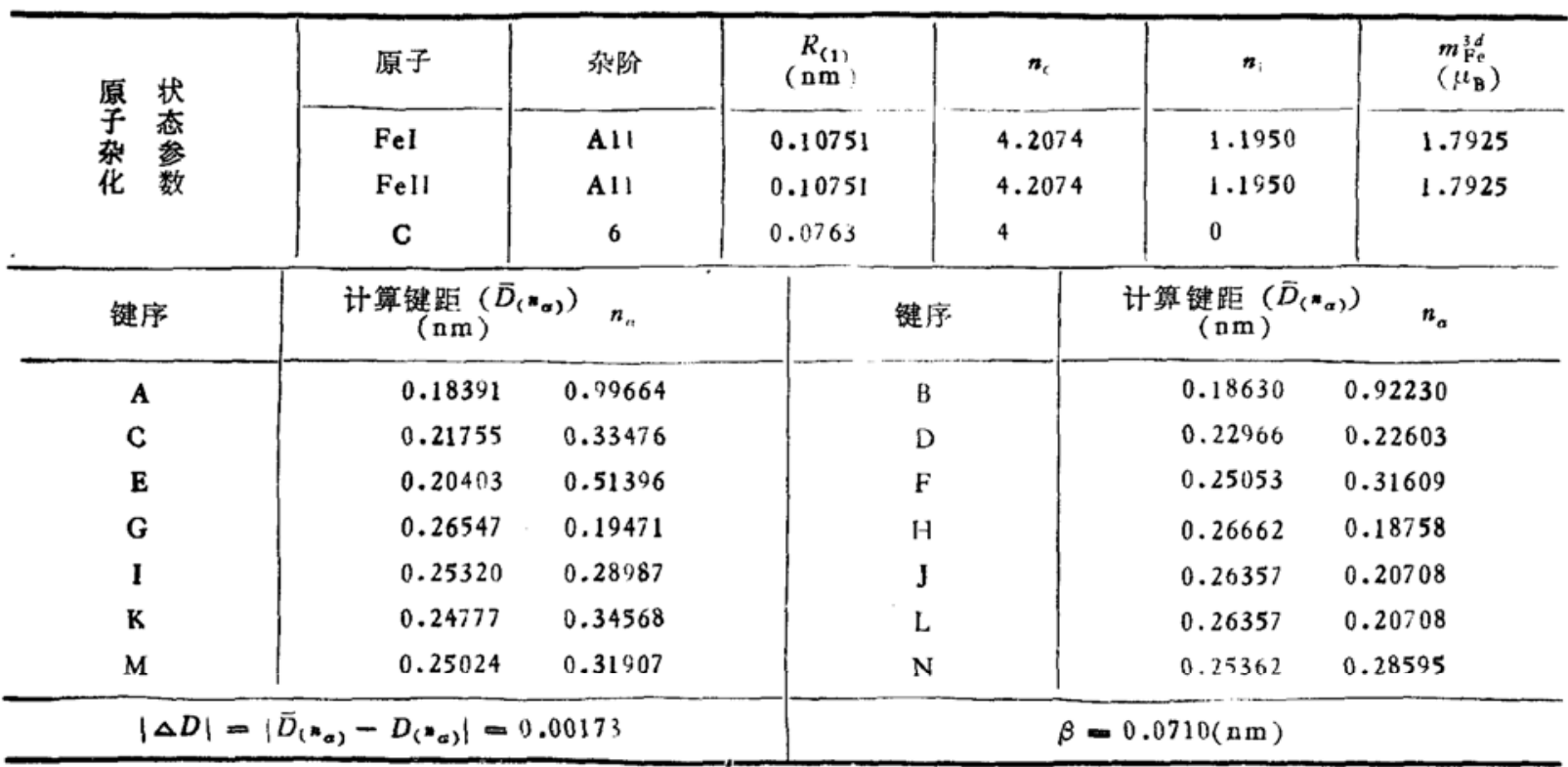

\section{3 价电子组成、 $\mathrm{Fe}$ 平均原子磁矩和晶格电子}

\section{1 各类原子的价电子组成}

由元素杂阶表 ${ }^{[4]}$ 可知: $\mathrm{FeI}(\mathrm{FeII}$ 同 $\mathrm{FeI}$ ) 原子为 $s-p-d$ 杂化, 价电子中含各类电子为

$$
\begin{array}{ll}
n_{\mathrm{s}} / n_{\mathrm{T}}=0.1997 ; & n_{\mathrm{p}} / n_{\mathrm{T}}=0.1250 ; \\
n_{\mathrm{d}} / n_{\mathrm{T}}=0.6753 & n_{\mathrm{s}}: n_{\mathrm{p}}: n_{\mathrm{d}}=2.0: 1.3: 6.8 .
\end{array}
$$

C 原子为 $s-p$ 杂化, 各类电子数量为:

$$
\begin{aligned}
& n_{\mathrm{s}} / n_{\mathrm{T}}=0.25 ; \quad n_{\mathrm{p}} / n_{\mathrm{T}}=0.75 ; \\
& n_{\mathrm{s}} / n_{\mathrm{p}}=1: 3 . \quad n_{\mathrm{T}} \text { 为总价电子数. }
\end{aligned}
$$

上列表明, $\mathrm{Fe}$ 中 $d$ 电子为主要成分, 这与 Häglund 等人结果一致. 


\subsection{Fe 的平均原子磁矩}

由表 1 , 渗碳体 $\mathrm{Fe}_{3} \mathrm{C}$ 中 $\mathrm{Fe}$ 的平均原子磁矩为 $1.7925\left(\mu_{\mathrm{B}}\right)$. 与 $\mathrm{LMTO}^{[2]}$ 的结果相比, 它更接近实验值 $1.72-1.78\left(\mu_{\mathrm{B}}\right)^{[2]}$.

\section{3 晶格电子与负磁矩}

由表 $1, \mathrm{Fe}$ 有 1.1950 个晶格电子, 按余瑞璜的分析 ${ }^{[3]}$, 一个晶格电子可贡献约 $-0.1684 \mu_{\mathrm{B}}$ 的磁矩, 可估计出在 $\mathrm{Fe}$ 三棱柱配位体的间隙处 (碳原子处), 由晶格电子引起之负磁矩约为 $-0.12 \mu_{\mathrm{B}}$. 这与 Häglund 等结果基本一致.

\section{4 渗碳体 $\mathrm{Fe}_{3} \mathrm{C}$ 的结合能}

徐万东等 ${ }^{[6]}$ 用余氏理论 ${ }^{[3]}$ 对渗碳体 $\mathrm{Fe}_{3} \mathrm{C}$ 的结合能作了计算, 结果为 $\bar{E}_{\mathrm{c}}-4^{\prime} / 9.8 \mathrm{~kJ} / \mathrm{mol}$, 与 Häglund 等人 ${ }^{[2]}$ 引用的实验值 $E_{\iota}=485.51 \mathrm{~kJ} / \mathrm{mol}$ 间的偏离仅为 $\sim 1.2 \%$, 这比 LMTO 的结果 $\bar{E}_{\mathrm{CH}}-807 \mathrm{~kJ} / \mathrm{mol}$ 要好得多.

\section{5 渗碳体 $\mathrm{Fe}_{3} \mathrm{C}$ 的键络和形貌}

渗碳体 $\mathrm{Fe}_{3} \mathrm{C}$ 常显片状特征, 这与其晶体键络特点有关. 图 1 示出了以 $\mathrm{C}$ 原子为中心的 $\mathrm{Fe}-\mathrm{C}$ 三棱柱配位体团. 它包括了晶体的全部最强键和绝大部 分次强键，可看作是最强键络基元. 这种基元连贯地排列形成， 了强键键络. 图 2 示意绘出了沿 $(a, c)$ 面强键键络的分布.

由图 2 可见, 沿 $(\boldsymbol{a}, \boldsymbol{c})$ 面, 晶体强键键络旺连续分布. 图 3 示出了沿 $(\boldsymbol{b}, \boldsymbol{c})$ 平面的键络分布, 由图, 沿 $\boldsymbol{b}$ 方向强键络不 是连续的, 有强键层带与弱键层带交替出现的特点. 图中影 线示出了弱键层带. 通过弱键连接着两个强键层的只有 $\mathrm{M}$ 键

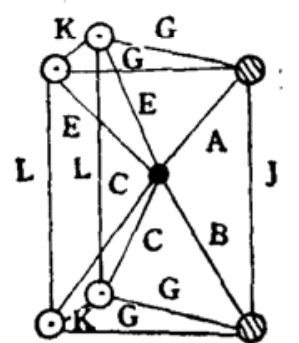

图 $1 \mathrm{Fe}-\mathrm{C}$ 棱柱配位体团 (2) $\mathrm{Fel}, 0$ 为 Fell, - 为C $\left(n_{\mathrm{M}}=0.31907\right)$.

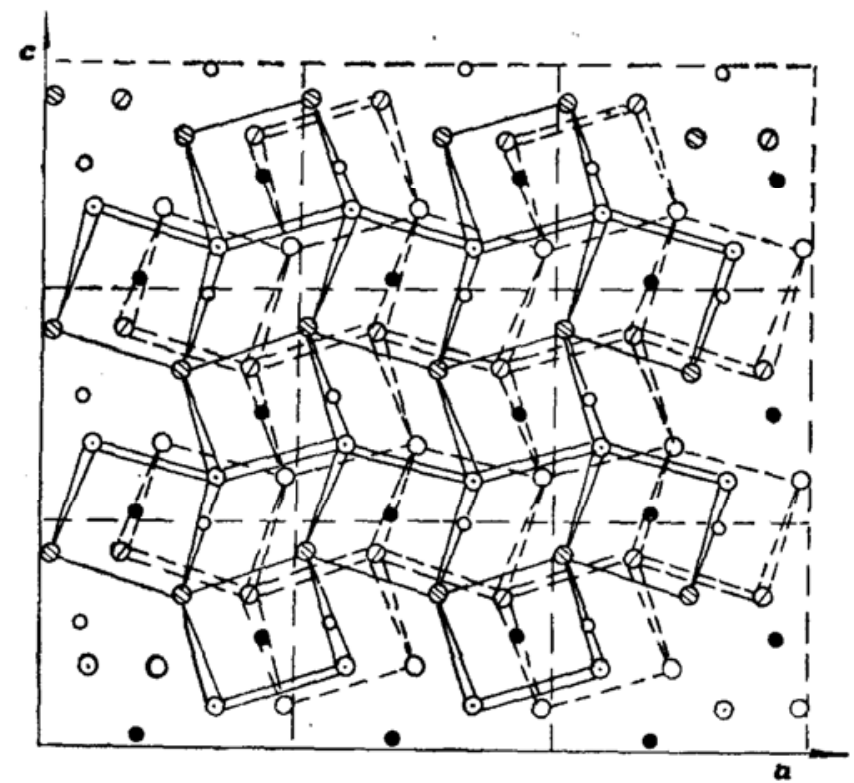

图 2 沿 $(a, c)$ 平面的强键键络

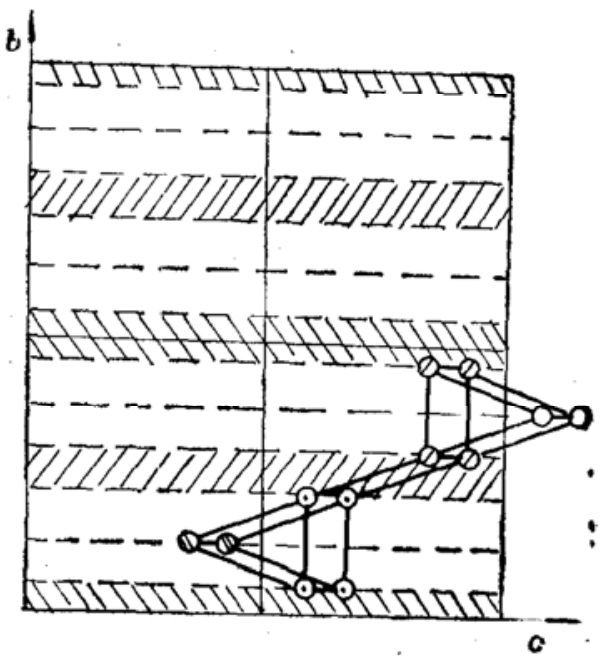

图 3 沿 $(b, c)$ 平面键络层带

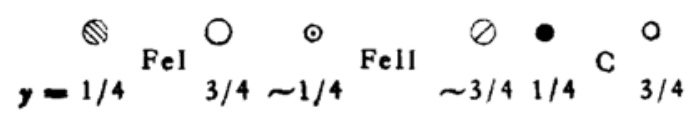


上述键络特点表明, 沿 $\boldsymbol{b}$ 方向原子堆积容易在弱键层处中断, 而沿 $(\boldsymbol{a}, \boldsymbol{c})$ 面由于强键络 的连续分布, 原子堆积不易中断. 这直观地说明了渗碳体 $\mathrm{Fe}_{3} \mathrm{C}$ 晶体呈片状形貌的本质原因.

\section{- 6 力 学 性 质}

参碳体 $\mathrm{Fe}_{3} \mathrm{C}$ 既硬又脆, 这种特点源于键的强度与共价电子(键络)分布的不均匀性. $n_{\mathrm{A}}$ 和 $n_{\mathrm{B}}$ （都 $>0.9$ ) 很接近共价单键, 有强共价键特性, 金属性弱, 故晶体有高硬度; 晶体键络在三 维方向的不均匀分布则是导致晶体脆性的主要原因 ${ }^{[n]}$.

\section{7 总 结}

余氏理论对参碳体 $\mathrm{Fe}_{3} \mathrm{C}$ 的某些计算结果与 $\mathrm{LMTO}$ 一致: $\mathrm{Fe}$ 原子为 $s-p-d$ 杂化, $d$ 电 子占主要部分; $c$ 为 $s-p$ 杂化; 碳原子处有负磁矩.

某些结果优于 L,MTO： $\mathrm{Fe}$ 的原子磁矩 $\bar{m}_{\mathrm{pe}}$ 值, 结合能 $\overline{\boldsymbol{E}}_{\mathrm{c}}$ 值都更接近实验值. 我们对键 络特点分析更解释了晶体形貌和力学性质的特点

\section{参考文 献}

[1] Lipson, H., Petch, N. J., J. Iron and Steel Inst., 1992, 105: 241.

[2] Haglund, J., Grimvall, G., Phys. Rev., 1991, 44: 2914.

[3] 余瑞璜,科学道报, $1978,23(3): 217$.

[4]张瑞林,余氏固体与分子经验电子理论蕉础, 吉林科技出版社, 1992.

[5] 余瑞璜,金属学报, 1982, 18: 336 .

[6] 徐万东、张瑞林、余瑞璜, 中国科学, A 辑, 1988, (3): 323.

[7] 王沿东、孙祖庆、蔡 军等,科学通报, 1991, 36(22): 1899. 